\title{
Hipótese da preservação de elos locais: uma explicação unificada para os déficits de produção e compreensão no agramatismo ${ }^{1}$
}

\author{
The local links preservation hypothesis: a unified \\ account for the production and comprehension \\ deficits in agrammatism
}

\section{Ricardo Joseh Lima Universidade Estadual do Rio de Janeiro}

\begin{abstract}
The goal of this paper is to discuss the relevance of studies of aphasic syndromes to linguistic theory. This will be done by analyzing the main features involving the definition and the work with agrammatism. Two main ideas will be defended: that agrammatism is an aphasic syndrome in which both linguistic production and comprehension are affected and that there are regular observable patterns in the disruption of these two modalities. It will be argued that these ideas can serve as the basis for the elaboration of a hypothesis that accounts in a unified manner for the deficits observed in agrammatism. This hypothesis, named "Preservation of Local Links", claims that only links that are local in grammar are preserved in agrammatism, while any other kind of non-local link is to be considered disrupted. In this way, the presentation of such hypothesis is a contribution to the dialogue that studies of aphasic syndromes can entertain with linguistic theories.
\end{abstract}

Keywords

Agrammatism; Minimalist Program; Neurolinguistics 


\section{Resumo}

O objetivo deste artigo é discutir a relevância de estudos de síndromes afásicas para a teoria lingüística. Isso será feito através da análise das principais características que envolvem a definição e o trabalho com o agramatismo. Duas idéias principais serão defendidas. Primeiro, que o agramatismo é uma síndrome afásica em que tanto a produção quanto a compreensão estão afetadas; segundo, que existem padrões regulares observáveis no comprometimento dessas duas modalidades. Será argumentado que essas idéias podem servir como a base para a elaboração de uma hipótese que dê conta de modo unificado dos déficits observados no agramatismo. Esta hipótese, chamada de "Preservação dos Elos Locais", afirma que somente elos que sejam locais na gramática estão preservados no agramatismo, enquanto que qualquer outro tipo de elo não-local estará afetado. Desse modo, a apresentação de uma hipótese como essa é uma contribuição para o diálogo que estudos de síndromes afásicas podem realizar com teorias lingüísticas.

\section{Palavras-chave}

Agramatismo; Programa Minimalista; Neurolingüística 


\section{INTRODUÇÃO}

"First, there is a faculty of language FL, a component of the buman mind/brain dedicated to language.(...) The language $L$ includes a cognitive system that stores information: roughly, information about sound, meaning, and structural organization. Performance systems access this information and put it to use. (...)We therefore take $L$ to be a cognitive system alone." (CHOMSKY, 2000). ${ }^{2}$

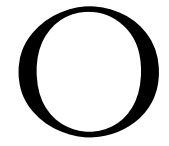

s estudos de afasias podem ser vistos como uma importante ferramenta na construção de teorias lingüísticas. Grodzinsky (1990) sugere que o critério da "compatibilidade de perda" seja acrescentado, ao lado dos critérios de aprendizagem, processabilidade e usabilidade propostos por Chomsky (1986), para avaliar teorias lingüísticas. Isso significa que, se uma teoria não conseguir prever os padrões de perda em uma determinada afasia, essa falha pode ser considerada equivalente à falha de, por exemplo, não conseguir dar conta de um determinado padrão no processo de aquisição.

Apesar desse objetivo ambicioso, os estudos de afasia vêm sendo pouco explorados em alguns trabalhos, que pouco citam dados vindos dessa fonte. Isso se deve a, pelo menos, dois fatores. O primeiro é o provável desconhecimento de que esse trabalho tem como foco não o sistema lingüístico do indivíduo afásico, mas o sistema lingüístico do indivíduo normal. Tal escolha se justifica pela observação de que a lesão cerebral modificou um sistema lingüístico já existente e qualquer caracterização do sistema pós-lesão será feito a partir do sistema lingüístico neurologicamente intacto. Assim, os estudos de afasia estabeleceriam de fato um "diálogo" com as teorias lingüísticas, como aponta Grodzinsky (1985). De um lado, inicia-se 
a descrição da síndrome afásica a partir de uma proposta de um sistema lingüístico do indivíduo normal; de outro, faz-se dessa descrição um meio de corroborar, desenvolver ou rejeitar a proposta de sistema que foi tomada como base. ${ }^{3} \mathrm{O}$ segundo fator que torna os estudos de afasias tão pouco "visíveis" é a pouca confiabilidade dos dados que são obtidos. Enquanto questões essenciais, como a própria definição do que vem a ser uma síndrome afásica ou como a validade da realização de estudos com grupos de afásicos (ver a discussão iniciada por Badecker \& Caramazza, 1985), ainda estiverem por ser resolvidas, a área caminhará com dificuldades. Outro fator que afeta a confiabilidade dos dados é o grau de variação encontrado nos estudos sobre uma mesma síndrome. Por exemplo, é possível justificar que a sintaxe está afetada no agramatismo (GRODZINSKY \& FINKEL, 1998) ou não (LINEBARGER et al., 1983) a partir da mesma fonte metodológica, a saber, o teste de julgamento de gramaticalidade. Claro está que qualquer área de investigação possui, por definição até, pontos conflitantes, divergências, resultados opostos, etc. Na área de estudos de afasia, no entanto, o sentimento de estar caminhando em "campo movediço" parece ser mais evidente. ${ }^{4}$

Nos últimos anos, tem-se tentado estabelecer alguns padrões para a síndrome afásica do agramatismo que se assentem em bases metodológicas plenamente justificáveis. Como conseqüência, tornase possível avançar no "diálogo" a ser estabelecido com as teorias lingüísticas do indivíduo normal. Especificamente, defende-se a idéia de que o agramatismo envolve déficits na compreensão e na produção lingüísticas e a idéia de que cada uma apresenta um padrão regular de perda. A apresentação dessas idéias é o foco da Seção 2.

A partir do estabelecimento dessas idéias, a possibilidade de construção de descrições do agramatismo que contribuam para o entendimento do sistema lingüístico do indivíduo normal se torna mais concreta. Na seção 3, uma dessas possibilidades, a saber a de Grodzinsky (1995, 2000a) será analisada. Dessa análise, surgirá, ainda na Seção 3, um questionamento sobre a natureza dos déficits no agramatismo. Será defendida a idéia de que uma explicação unificada 
para os déficits é possível. A implementação dessa idéia será feita na Seção 4, na qual também se discutem as vantagens e desvantagens de tal idéia. A Seção 5 encerra o artigo ressaltando os temas gerais dos estudos de afasias para as teorias do sistema lingüístico do indivíduo normal.

\section{A REGULARIDADE NA SÍNDROME AFÁSICA DO AGRAMATISMO}

Desde pelo menos Badecker \& Caramazza (1985), dois desafios vêm sendo lançados para os estudiosos do agramatismo. ${ }^{5}$ Esses desafios colocam em xeque a própria existência de uma síndrome chamada agramatismo. Isso porque os autores mencionados e outros (MARTIN et al., 1989; BERNDT et al., 1996) argumentam que as características seguintes não são encontradas em todos os pacientes classificados como agramáticos:

(a) co-ocorrência de problemas de produção e de compreensão;

(b) padrões regulares de perda no desempenho lingüístico.

A primeira característica vem se contrapor a visões como a de Caramazza \& Zurif (1976) e Grodzinsky (1984), por exemplo, de que todo agramático apresenta simultaneamente problemas nas duas modalidades citadas. Os casos de interesse são os de agramáticos com problemas de produção (a característica intrínseca dessa síndrome), mas sem problemas de compreensão. As fontes mais citadas são os trabalhos de Miceli et al. (1983), Kolk \& van Grusven (1985), Nespoulous et al. (1988) e Martin et al. (1989). Em todos esses trabalhos são descritos pacientes agramáticos que não apresentam problemas de compreensão.

A segunda característica é apontada já no trabalho de Badecker \& Caramazza (1985), que cita resultados desencontrados da literatura (MICELI et al., 1983; CAPLAN et al., 1985; SAFFRAN et al., 1980). Especificamente, os padrões encontrados em um estudo não são replicados em outro. Assim é que os pacientes de Saffran et al. (1980) 
têm problemas com a morfologia flexional, mas os de Miceli et al. (1983) não. Por outro lado, há casos em que a omissão de verbos é freqüente, enquanto, em outros, a omissão de verbos não é significativa. O estudo de Berndt et al. (1996) pretendeu dar um tratamento estatístico a um número significativo de estudos com pacientes agramáticos a fim de demonstrar que um determinado padrão na compreensão, a saber, bom desempenho na compreensão de sentenças ativas, mas não de sentenças passivas, não seria encontrado em mais do que um terço dos agramáticos.

Ambos os desafios colocados vêm sendo respondidos em várias frentes. Caplan (1986) foi o primeiro a observar que a variação deveria ser encarada como um traço usual de uma síndrome afásica e não como algo exterior a ela, o que seria problemático. A solução apontada seria trabalhar a análise dessa variação e não tomá-la como empecilho para a definição da síndrome do agramatismo.

Em relação ao desafio (a), Caplan (1991), Grodzinsky (1991), Swinney \& Zurif (1995), entre outros, fazem uma análise criteriosa dos pacientes que foram estudados por Miceli et al. (1983), Kolk \& van Grusven (1985) e Martin et al. (1989). A conclusão a que chegam é que muitos desses pacientes não são agramáticos, por dois motivos (isolados ou não): o local da lesão não era mencionado ou não era a área de Broca; e a produção lingüística dos pacientes não se encaixava como sendo agramática, ou seja, telegráfica, com o predomínio de palavras de "conteúdo" (itens substantivos, como nomes, verbos e adjetivos) e sem itens funcionais (como preposições, artigos, morfemas flexionais, etc.).

O segundo desafio, o da variação, (b), também foi respondido (PIÑANGO, 1998; ZURIF \& PIÑANGO, 1999; DRAI \& GRODZINSKY, 1999, entre outros). Um dos argumentos das respostas era o de analisar criteriosamente a ferramenta estatística para se calcular o que era variável e o que era regular. No entanto, o argumento central focalizava a escolha dos pacientes que entravam no cálculo estatístico feito por Berndt et al. (1996). Pacientes sem o local da lesão definido e sem uma fala agramática eram incluídos enquanto pacientes com 
lesão na área de Broca e com fala agramática eram excluídos. Berndt et al. (1996), bem como Caramazza et al. (2001) e Druks \& Marshall (2001) argumentavam que esses critérios (local da lesão e habilidades na produção) não eram determinantes na escolha e que a exclusão se devia à ausência de informações seguras sobre os pacientes que poderiam se encaixar no perfil do agramático.

Como se pôde ver, a questão da seleção de pacientes está presente nos desafios e nas respostas. Quando do artigo de Badecker \& Caramazza (1985), a área de estudos em afasia ainda se encontrava bastante incipiente nessa questão. Quase duas décadas depois, no entanto, já é possível listar critérios que venham, se não a constituir um paradigma para a seleção de pacientes, pelo menos, a servir de base para comparação entre estudos. Desse modo, por exemplo, dois estudos podem ser comparados se os pacientes em ambos os estudos tiveram sido selecionados pelos mesmos critérios. Uma série de artigos (MAUNER, 1995; CAPLAN, 1995; KEAN, 1995) foi publicada com a finalidade de discutir essa questão e apontar caminhos para a sua solução. Dentre esses caminhos, podem ser citados como critérios que deveriam ser considerados consensuais:

(i) conhecimento do local da lesão;

(ii) descrição do desempenho do(s) paciente(s), comparando-se com outros na literatura;

(iii) aplicação dos testes, quando houver, em indivíduos normais e nos afásicos em uma quantidade que seja estatisticamente significativa.

De posse desses critérios, é possível selecionar um determinado conjunto de afásicos e analisar somente para esse conjunto os desafios de co-ocorrência de sintomas e de variação. Essas análises foram realizadas em trabalhos como o de Piñango (1998) e Drai \& Grodzinsky (1999). Trata-se, portanto, de uma linha de pesquisa que toma a variação como característica inerente de afasias (Cf. SANCHEZ, 1996) e que, adotando determinados critérios, é capaz de argumentar 
contra casos extremos de variação. Por fim, essa linha de pesquisa pretende tratar as exceções (Cf. o estudo de NESPOULOUS et al., 1988) como tais e não como balizadoras de uma nova definição de uma síndrome. Em outras palavras, assume-se a postura metodológica de não começar pelo pior caso nem fazer dele um elemento central de preocupação, e a postura de tentar encontrar análises adequadas para aquilo que é regular. A respeito desse último ponto, Grodzinsky (1984) salienta as semelhanças encontradas na produção lingüística de agramáticos de várias línguas, como inglês, italiano, hebraico e russo e enfatiza a decisão de se procurar uma explicação para essa regularidade.

Lima $(2003)^{6}$ adotou os critérios (i) a (iii) apresentados para avaliar estudos com afásicos considerados como agramáticos. Desse conjunto, foram extraídos vários subconjuntos utilizando-se um critério comparativo para aferir a confiabilidade dos padrões encontrados. Assim, um padrão de desempenho encontrado em vários estudos deve receber um tratamento diferenciado, no caso privilegiado, de um padrão confirmado por apenas um estudo. Em uma escala "inferior" estaria o padrão observado apenas uma vez e, por fim, um padrão que, tendo sido observado apenas uma vez, foi refutado em estudo posterior.

A decisão de adotar os critérios (i) a (iii) e o da confiabilidade dos dados pareceu condição sine qua non para a construção de uma descrição adequada da síndrome afásica do agramatismo, como se verá na Seção 4. Tal decisão está de acordo com os artigos citados de Mauner, Caplan e Kean, refletindo as preocupações neles emitidas. Não se pretendeu que os critérios relacionados fossem considerados como consensuais ou os mais adequados. Apenas foi a intenção ressaltar a importância da necessidade extrema de que esses critérios sejam levados em conta; caso contrário, a área de estudos de afasias corre o risco de se tornar uma discussão infantilizada. ${ }^{7}$ 


\section{A NATUREZA DOS DÉFICITS NO AGRAMATISMO}

Grodzinsky é um dos estudiosos que constrói suas hipóteses para uma síndrome afásica a partir da escolha metodológica mencionada anteriormente, ou seja, a de observar as regularidades da síndrome intra e entre pacientes. Nesta seção, será feita uma análise das hipóteses de Grodzinsky sob a ótica da natureza dos déficits. Essa análise levará a uma discussão que possibilitará a formulação da Hipótese da Preservação de Elos Locais.

O primeiro trabalho de Grodzinsky sobre o agramatismo (1984) ainda está sob a influência da hipótese de Caramazza \& Zurif (1976). De acordo com essa hipótese, o agramatismo era uma síndrome afásica que afetava a compreensão e a produção lingüísticas e os problemas observados nessas modalidades eram o resultado da incapacidade dos agramáticos de operar algoritmos sintáticos. A hipótese de Caramazza \& Zurif (1976), por ter a característica de tentar explicar os dois déficits a partir de uma única fonte, foi chamada de overarching (aqui "englobadora"). Antes de iniciar a exposição das hipóteses de Grodzinsky, vale a pena nos determos na história dessa hipótese englobadora.

A motivação para o surgimento de uma hipótese englobadora vem da reação de alguns estudiosos à distinção proposta entre Competência e Desempenho feita por Chomsky (1965). Entre os vários argumentos que, na época, foram elaborados contra tal distinção, um veio da área de estudos de afasias. Whitaker (1970) observou que não havia nenhuma síndrome afásica que afetasse mais de uma modalidade, entre compreensão e produção. Ora a produção era afetada (agramatismo, afasia de Broca), ora a compreensão (afasia de Wernicke). O que se deveria esperar da existência de um sistema que servisse às duas modalidades e que estivesse localizado no cérebro (a Competência) é que alguma lesão o afetaria, resultando em um déficit que atingisse tanto produção quanto compreensão. Como tal perfil de déficit não era observado, a existência da Competência era colocada em xeque. Caramazza \& Zurif (1976), entre outros, demonstraram que agramáticos, além de possuírem 
problemas de produção, também possuem problemas, mais restritos, de compreensão. O "desafio" lançado por Whitaker (1970) estava, então, respondido. Com isso, era possível propor uma descrição de uma afasia cuja causa seria uma alteração na Competência. Desse modo, seria possível extrair uma única fonte (no caso de Caramazza \& Zurif, o algoritmo sintático) para dar conta dos dois déficits.

Grodzinsky (1984) propõe sua primeira hipótese baseado no cenário apontado pela frase anterior. Para explicar por que agramáticos possuíam problemas na produção de morfemas flexionais, artigos e preposições e problemas na compreensão com a categoria vazia vestígio, Grodzinsky propôs que os nós não-lexicais ficassem subespecificados na Estrutura-S na gramática do agramático. Com isso, no caso da produção, o preenchimento desses nós, forçado por condições de boa-formação fonológica, se daria de modo aleatório; no caso da compreensão, a subespecificação dos vestígios resultaria em uma incapacidade na transmissão de papel temático, gerando problemas de interpretação.

Embora tivesse sido uma hipótese englobadora, a primeira hipótese de Grodzinsky não se comprometia com a fonte do déficit. Assim, a Estrutura-S é escolhida para ser o local de origem dos déficits porque Grodzinsky considera que esse nível realiza a interface entre o sistema lingüístico e os sistemas de processamento. Em trabalhos posteriores (Grodzinsky 1986, 1990, 1995) e até nos mais recentes (2000a), Grodzinsky deixa em aberto a questão de os déficits terem origem no sistema lingüístico ou nos sistemas de acesso a ele, os sistemas de processamento.

Entretanto, como se pode notar, sustentar a idéia de que os déficits têm origem nos sistemas de processamento parece ser incompatível com a idéia da hipótese englobadora. De fato, o que fez Grodzinsky sempre deixar como opção a interpretação de a origem dos déficits ser nos sistemas de processamento foi a observação de que os perfis de desempenho em cada modalidade pareciam não possuir uma origem comum. Isso se deve ao desenvolvimento da área de estudos de afasias, que foi revelando, 
aos poucos, detalhes dos desempenhos dos agramáticos na produção e na compreensão.

Pelo lado da produção, observou-se que os agramáticos possuem mais problemas com elementos situados nos nós mais altos da árvore sintática (HAGIWARA, 1995; FRIEDMANN \& GRODZINSKY, 1997). Assim, questões envolvendo elementos QU- e sentenças envolvendo movimento de verbos para $\mathrm{C}$ ou $\mathrm{T}$ seriam mais problemáticas, pois envolvem as posições mais altas da árvore. Já as relações de concordância e as de Caso podem ser estabelecidas no âmbito de T e Agr e até dentro do VP (como na designação de Caso acusativo, por exemplo). Essas são as posições mais baixas na árvore sintática e, por isso, essas relações se encontram menos afetadas na produção agramática. Grodzinsky propõe, juntamente com Friedmann (FRIEDMANN \& GRODZINSKY, 1997), que o problema na produção agramática esteja na indisponibilidade seletiva (a depender da severidade da lesão) das categorias funcionais. Note-se que essa hipótese tanto pode ser vista como relacionada ao sistema lingüístico (as categorias funcionais não estão mais disponíveis; aconteceria o inverso da aquisição, em que as categorias funcionais parecem ser disponibilizadas aos poucos) como ao sistema de processamento (seria mais custoso em termos processuais acessar as categorias funcionais mais altas).

Pelo lado da compreensão, os estudos feitos (HICKOK et al. 1993; SADDY, 1995; HICKOK \& AVRUTIN, 1995; GRODZINSKY \& FINKEL, 1998) indicam que estruturas que contêm movimento aberto de sintagma, ou seja, antes da Estrutura-S, são problemáticas para os agramáticos. Grodzinsky mantém nos trabalhos posteriores (1995, 2000a) a idéia apontada no primeiro trabalho de que os vestígios estão, de algum modo, inacessíveis na "gramática" do agramático. Mais uma vez, o modalizador "de algum modo" revela a dupla possibilidade da origem do déficit: os vestígios podem estar apagados ou mesmo não serem produzidos (Cf. NOVAES, 2000), o que levaria o déficit a se originar no interior do sistema lingüístico, ou o acesso aos vestígios no processamento on-line poderia estar rompido, 
devido ao fato de essa operação ser custosa para o sistema de processamento, que seria a origem do déficit.

As hipóteses de Grodzinsky sobre produção e compreensão são consideradas como parâmetro para a formulação de novas hipóteses, se o arcabouço utilizado for o da Teoria Gerativa (HICKOK et al., 1993; MAUNER et al., 1993; BERETTA et al., 1996; PIÑANGO, 1998, entre outros). Em relação à hipótese sobre a compreensão agramática, o próprio Grodzinsky a classifica apenas como uma entre as possiveis descrições do que deve, provavelmente, ser o âmago do déficit: o movimento aberto de sintagmas. Grodzinsky opta por se concentrar no vestígio e pelo termo "apagamento", embora sempre se tenha deixado claro que uma hipótese baseada em cadeias ou que use o termo "inacessibilidade" seria igualmente capaz de capturar os fatos da compreensão agramática.

Entretanto, as hipóteses de Grodzinsky, como mencionado, apenas seguiram uma das propostas de Caramazza \& Zurif (1976), a saber, a de que o agramatismo envolve déficits de compreensão e de produção simultaneamente. A outra proposta, que caracteriza o trabalho de Caramazza \& Zurif como "englobador", de que a origem desses déficits deve ser única, não é incorporada pelas hipóteses de Grodzinsky. Desde pelo menos seu trabalho de 1990, Grodzinsky observara que os padrões superficiais de desempenho não eram idênticos nessas modalidades. Assim, enquanto a produção de sentenças com movimento de verbo é problemática, a compreensão dessas sentenças não é; a compreensão de violações nos morfemas de tempo parece estar intacta, ocorrendo o oposto na produção desses morfemas. Essas observações levaram Grodzinsky (1990, 1995) a formular hipóteses distintas para os déficits de produção e compreensão, a fim de dar conta separadamente de cada um deles.

Tal abordagem é reafirmada em trabalho posterior, (GRODZINSKY, 2000a), em que Grodzinsky propõe que a área de Broca não é o local da "linguagem articulada", mas apenas das transformações sintáticas envolvendo sintagmas. Tal concepção é claramente baseada na hipótese sobre a compreensão. Para acomodar a hipótese sobre a 
produção, Grodzinsky propõe que a área de Broca também seja a sede da construção da árvore sintática. Entretanto, essa acomodação traz pelo menos dois problemas: o primeiro é que a uma mesma área são estipuladas funções distintas; o segundo, talvez mais crucial, é que, mesmo com essa dupla função, a área de Broca lesionada resulta déficit de compreensão apenas relacionado ao movimento de sintagmas e déficit de produção somente relacionado à construção da árvore sintática. Em tese, o déficit de compreensão poderia ser relacionado à construção da árvore sintática e o da produção ao movimento de sintagmas, visto que a área lesionada possuiria essa dupla função. No entanto, tais perfis de desempenho não são observados.

Em outro trabalho, Grodzinsky (2000b) observa que a hipótese englobadora não pode ser descartada. Para ele, apenas falta um instrumental teórico que consiga sustentar uma hipótese unificada para os déficits no agramatismo. Nesse caso, a hipótese englobadora de Caramazza \& Zurif seria redimida, depois de tantos anos sendo colocada em xeque. Note-se que, se tal hipótese puder ser formulada, os dois problemas apresentados no parágrafo anterior perdem sentido: a área de Broca ficaria com apenas uma função e a alteração dessa função resultaria nos déficits observados na compreensão e na produção. A seção a seguir apresenta uma proposta de hipótese englobadora para o agramatismo.

\section{AGRAMATISMO, UMA AFASIA ENGLOBADORA}

Há pelo menos quatro argumentos indiretos que favorecem uma abordagem englobadora para o agramatismo. Dois desses argumentos já foram vistos. O primeiro é o "paradoxo" de Whitaker revisto: se a Competência é parte da arquitetura da mente/cérebro, então ela deve estar sujeita à alteração devido a danos cerebrais. Esse argumento será detalhado logo a seguir. O segundo argumento diz respeito à função da área de Broca na linguagem: tendo uma função única, uma lesão nessa área resultará em alterações na compreensão e na produção. 
Um terceiro argumento indireto é o estatuto de "candidato natural" a déficit que afete o sistema lingüístico (e não os sistemas de processamento e desempenho) que o agramatismo apresenta. A síndrome afásica do agramatismo é a única que apresenta em um grande número de pacientes alterações na produção e na compreensão simultaneamente. Afásicos de Wernicke, por exemplo, são fluentes apesar de não possuírem compreensão lingüística; afásicos de condução são incapazes de repetir palavras e sentenças, mas sua compreensão lingüística permanece inalterada.

Por fim, um quarto argumento indireto seria o caráter sintático dos déficits no agramatismo. Grodzinsky argumenta consistentemente que o nível sintático é o nível de análise mais adequado para essa síndrome (Grodzinsky 1990, Friedmann \& Grodzinsky 1997). Se levamos em conta uma visão chomskyana de que o sistema lingüístico se resume a um sistema computacional $\left(\mathrm{C}_{\mathrm{HL}}\right)$ que apenas realiza operações sintáticas, então pode-se levantar a hipótese de que no agramatismo algo desse sistema esteja afetado.

Essa idéia retoma o primeiro argumento. Se o trecho citado na Introdução está correto, ou pelo menos faz sentido a partir do entendimento da relação entre a Faculdade de Linguagem e os demais sistemas cognitivos, então, mais do que viável, torna-se até desejável que se construa uma hipótese sobre uma síndrome afásica. Tal hipótese se basearia na premissa de que esse sistema cognitivo que é a Faculdade da Linguagem e, visto de modo mais restrito, apenas o sistema computacional $\left(\mathrm{C}_{\mathrm{HL}}\right)$, possa vir a ser alterado por uma lesão cerebral.

A hipótese englobadora a ser formulada, portanto, deve visar a esse sistema computacional, propondo uma alteração, da qual resultarão os padrões de perda na produção e na compreensão lingüísticas no agramatismo. A hipótese de Grodzinsky para a compreensão pode servir de base para a hipótese englobadora: movimentos abertos de sintagmas são problemáticos para os agramáticos. Como visto na Seção 3, movimentos abertos não parecem ser o cerne do problema na produção agramática, embora 
claramente eles também sejam problemáticos, dada a dificuldade dos agramáticos em produzir sentenças com elemento QU- deslocado. O problema na produção lingüística parece ser o da relação entre os traços das categorias funcionais e os das categorias lexicais (nome, verbo, etc.). O que parece unir essas duas descrições dos déficits no agramatismo é o fato de que, em ambas, há uma relação entre duas posições na árvore sintática: no caso da compreensão, entre o sintagma movido e sua cópia; no caso da produção, entre uma categoria e outra.

Observando dois casos simples como "João $v$ viu Maria" e "Que menino ${ }_{i}$ o João viu v?", percebemos que essas relações entre posições podem variar em termos de localidade. No primeiro caso, a relação seria estritamente local, sem nenhum elemento intervindo entre o elemento movido e seu vestígio; no segundo caso, o elemento QUse desloca cruzando um sintagma, o DP João. Sabendo que a primeira das duas sentenças apresentadas é tanto compreendida quanto produzida normalmente por um agramático, mas a segunda não é compreendida nem produzida com facilidade, pode-se supor que algo específico da relação entre duas posições na árvore sintática esteja envolvido no agramatismo. A escolha recai sobre o caráter de localidade dessa relação, entendendo-se por localidade a ausência de elementos intervenientes de determinado tipo entre as posições em questão.

Como mais de um tipo de relação está envolvido no agramatismo (sintagma movido e sua cópia, núcleo movido e sua cópia, categoria funcional e categoria lexical), é preferível utilizar o nome elo para o resultado do estabelecimento dessas relações. Assim, a Hipótese da Preservação de Elos Locais (LIMA, 2003) prevê que apenas os elos que são locais estejam intactos no agramatismo. Com isso, é possível dar conta da dificuldade dos agramáticos na compreensão de sentenças passivas como "O menino foi empurrado $v_{i}$ pelo homem". Entre o DP "o menino" movido e sua cópia, pode estar intervindo, dependendo da análise escolhida, o morfema de particípio como argumento (BAKER et al., 1989) ou uma categoria vazia representando esse argumento (BOECKX, 1998). 
No caso da produção de sentenças com elemento QU- deslocado, é possível adotar uma análise baseada em fases (CHOMSKY, 2000, 2001) para visualizar que a relação entre o elemento na sua posição final e na sua posição inicial não é local, ou pelo menos é "menos local" que a relação entre um DP deslocado para Spec T e sua posição original em Spec v. Isso também aconteceria com o verbo: o movimento de $\mathrm{V}$ para $\mathrm{v}$ ocorre dentro de uma fase (vP), mas o de $\mathrm{v}$ $\mathrm{V}$ para T é um movimento entre fases (v e C, que contém T). Essa distinção originaria a diferença na localidade dos elos: local para V-v, mas não para $[\mathrm{V}-\mathrm{v}]$ para T. Sem um elo local, a relação entre $\mathrm{V}$ em T e sua posição de origem fica prejudicada, resultando no fraco padrão de produção de morfemas de tempo, que são alocados de modo aleatório ao radical do verbo ("Amanhã, ele comeu") (FRIEDMANN \& GRODZINSKY, 1997).

Voltando aos desempenhos na compreensão, a Hipótese da Preservação de Elos Locais consegue dar conta da distinção entre sentenças relativas de sujeito "É o menino que empurra a menina" (bom desempenho) e relativas de objeto "É o menino que a menina empurra" (desempenho fraco). Apenas no segundo tipo de sentença existe um elo não-local. Esse tipo de elo não é tolerado pela gramática do agramático e, portanto, a compreensão da sentença fica prejudicada. Duas vantagens dessa hipótese podem ser vistas se comparada com a hipótese de Grodzinsky: primeiro, não faz menção à categoria vestígio (ou cópia). Com isso, não lhe atribui nenhum status especial no sistema (por que a cópia debaixo seria afetada, e não a de cima?), o que está de acordo com um tratamento nãodiferenciado para cópias (NUNES, 2004). Segundo, não faz uso de uma estratégia não-lingüística (o Princípio Default de Grodzinsky, 1986), que operaria seletivamente e apenas em certos casos.

Um dos desafios que se tem colocado à hipótese de Grodzinsky é o caso de sentenças com "movimento coberto", envolvendo "every DP" e "who" (SADDY, 1995; HICKOK \& AVRUTIN, 1995). Os dados relativos a essas sentenças não estão no mesmo nível de confiabilidade dos dados das sentenças nas vozes ativa e passiva e 
nas relativas de sujeito e objeto. Entretanto, se forem adotadas algumas das abordagens de "movimento coberto" (KITAHARA, 1996; CHOMSKY, 1995; PESETSKY, 1998) disponíveis, então se poderia pensar esse tipo de movimento resultando em um elo local, pelo menos nos casos em que o elemento a ser movido está em Spec C ou se desloca de Spec T para Spec C. A derivação contaria com um elo local e um elo não-local relacionando duas posições na árvore sintática. Adotando-se a idéia de Richards (1998), de que a satisfação a uma exigência pode mascarar para o sistema uma violação dessa exigência, pode-se supor que a existência de um elo local "salva" o elo não-local. Deve-se ressaltar, no entanto, que essa explicação só pode vir a ser utilizada se o padrão de desempenho com elementos que sofrem "movimento coberto" vier a ser confirmado por estudos posteriores.

Em situação semelhante, à espera de confirmação de outros estudos, encontram-se as passivas com verbos psicológicos. O padrão obtido até o momento confirma os insights de Belletti \& Rizzi (1988): sentenças como "O menino é assustado pela menina" não seriam passivas sintáticas, mas passivas adjetivas. ${ }^{8}$ Desse modo, não haveria movimento da posição de objeto e, portanto, não haveria elos não-locais; como conseqüência, o desempenho na compreensão dessas sentenças é bom. Além disso, a compreensão de sentenças na vOz ativa com verbos desse tipo ("O menino assusta a menina") é, curiosamente, inverso, ou seja, fraco. A análise de Belletti \& Rizzi fornece o instrumental para a explicação desse padrão: o DP "O menino" deve ter-se movido de alguma posição de complemento do VP. Esse movimento resultaria em um elo não-local (veja-se sua implementação em um arcabouço minimalista em Bennis, 2000), ocasionando a fraca compreensão desse tipo de estrutura.?

\section{CONCLUSÕES}

As explicações para os padrões de desempenho em sentenças com "movimento coberto" e com verbos psicológicos necessitaram de acomodações e da assunção de determinadas idéias. Isso não 
diminui a força da Hipótese da Preservação de Elos Locais pois, como foi observado, esses padrões ainda estão em fase de definição e podem vir a não ser confirmados em estudos posteriores. Também como observado anteriormente, a Hipótese da Preservação de Elos Locais não necessitou da estratégia não-lingüística do Princípio Default (sugerida por Grodzinsky (1984) e criticada por Hickok et al. (1993), Mauner et al. (1993) e Linebarger (1995), entre outros), de aplicação irregular ${ }^{10}$ para explicar os padrões consensuais de desempenho no agramatismo. Para finalizar, serão feitas três observações.

A primeira diz respeito a uma abordagem sobre os sistemas de processamento que considera os mecanismos internos a esses sistemas como sendo reflexos dos mecanismos internos do sistema computacional $\mathrm{C}_{\mathrm{HL}}$ (WEINBERG, 1999). Assim, os sistemas de processamento trabalhariam com os mesmos conceitos e operações propostos para o sistema computacional, por exemplo, Agree, SpellOut múltiplo, fases, etc. A adoção dessa abordagem pode tornar o tema da natureza dos déficits no agramatismo irrelevante, já que se estaria diante de um único sistema em dois momentos, estático $\left(\mathrm{C}_{\mathrm{HL}}\right)$ e dinâmico (processamento). Dentro de tal abordagem, os únicos argumentos para a natureza do déficit ser no momento estático e não no dinâmico seriam os argumentos indiretos mencionados na seção anterior.

A segunda observação é sobre uma explicação alternativa à Hipótese da Preservação de Elos Locais que se enquadre no arcabouço teórico do Programa Minimalista. Assumindo que os déficits têm origem no sistema computacional, uma possibilidade de explicação que não foi levantada seria identificar a causa dos déficits nas interfaces, PF e LF. Uma formulação geral como "Relaxe as condições de interface" poderia ser proposta. Tal formulação poderia ter duas vantagens: primeiro, poderia estabelecer uma escala de severidade no agramatismo, por exemplo, enumerando-se uma série de condições e, nos casos mais suaves, apenas algumas dessas seriam relaxadas; segundo, a formulação poderia dar conta dos casos em que 
há apenas problema de produção, mas não de compreensão: nesses casos, apenas as condições de PF estariam relaxadas, enquanto as de LF estariam mantidas.

No entanto, uma formulação como a sugerida enfrentaria alguns problemas. Em primeiro lugar, não há um conhecimento aprofundado de quais são as condições das interfaces; além disso, parece ser pouco provável que ambas as interfaces possuam as mesmas condições, dadas as características particulares de cada uma. A enumeração de condições seria problemática de um ponto de vista teórico, pois a idéia é que a alteração no sistema causada pela lesão deva ser mínima. ${ }^{11}$ Por fim, em relação à dissociação entre PF e LF, não há casos de agramáticos apenas com problemas de compreensão, mas sem problemas de produção, um quadro que seria previsto pela formulação apresentada.

Finalmente, a terceira observação diz respeito à contribuição que os estudos de afasias podem levar para os estudos do sistema lingüístico do indivíduo normal, retomando-se a discussão apresentada na Introdução. Nesse artigo, pretendeu-se ressaltar essa possibilidade de contribuição ao se tomar um quadro teórico definido (a Teoria Gerativa e, mais especificamente, o Programa Minimalista) para realizar a descrição de uma síndrome afásica. Com isso, além das discussões inerentes aos estudos de afasias (padrões de regularidade, seleção de pacientes, etc.), a hipótese aqui discutida suscitou reflexões sobre a arquitetura da Faculdade da Linguagem e suas relações com os demais sistemas cognitivos. Espera-se também que os temas levantados tenham feito surgir o interesse pela área de estudos de afasias, tão árdua quanto instigante. 


\section{NOTAS}

${ }^{1}$ Professor Adjunto da Universidade do Estado do Rio de Janeiro (UERJ). Este artigo se baseia na minha tese de doutorado, defendida em 2003. Agradeço à banca pelos comentários realizados. Uma versão anterior desse artigo circulou no encontro do GT de Teoria da Gramática, na USP, em dezembro de 2003. Agradeço aos participantes desse evento, especialmente a Maria Irma Hadler Coudry e Sergio Menuzzi, pelos comentários e a um parecerista pelas observações pertinentes. Todos os erros remanescentes são de minha responsabilidade.

2 "Em primeiro lugar, existe uma faculdade da linguagem FL, um componente da mente/cérebro humano(a) dedicado à língua (...) A língua L inclui um sistema cognitivo que armazena informação; em linhas gerais, informação sobre som, significado e organização estrutural. Sistemas de desempenho acessam essa informação e a colocam em uso (...) Portanto, consideramos L como sendo um sistema cognitivo por si só." (Tradução do autor.)

${ }^{3}$ É o que faz Grodzinsky (1990), ao comparar as análises da Teoria da Regência e Ligação e da Lexical Functional Grammar, a respeito de dois tipos de passivas (adjetivas e sintáticas) a partir de dados de estudos com agramáticos.

${ }^{4}$ Ver o título do artigo de Kean (1995): "The elusive character of agrammatism".

${ }^{5}$ A primeira definição de agramatismo o toma como sendo uma síndrome afásica cuja característica principal é a fala "telegráfica" resultante de lesão na área de Broca. Mais tarde se incorporou a informação de que alguns aspectos da compreensão lingüística estão afetados nessa síndrome. Um parecerista fez a interessante observação de que o termo, tal como está, indica ausência de gramática (a-gramática), o que contradiz sua definição atual, de ser o agramatismo um déficit seletivo. Deve-se esclarecer que há consciência desse descompasso entre o termo e a descrição da síndrome; o termo continua a ser usado, no entanto, por ser tradicional e já amplamente conhecido.

${ }^{6}$ Um parecerista observou que essa é a única referência em português do artigo, acrescentando que há trabalhos realizados na Unicamp e na PUC-SP sobre afasia e agramatismo. Não há desconhecimento desses trabalhos, nem se coloca em questão sua importância para a área. Apenas não são mencionados nesse artigo por se tratar de trabalhos que utilizam uma vertente teórica distinta da utilizada aqui.

${ }^{7}$ Ver os títulos dos artigos de Caramazza et al. "Agrammatic Broca's aphasia is not associated with a single pattern of comprehension" e de Drai \& Grodzinsky "Broca's aphasia is associated with a single pattern of comprehension performance: A reply". 
${ }^{8}$ Passivas sintáticas e passivas adjetivas se diferem por se considerar que, no primeiro tipo ("O menino foi empurrado $v_{i}$ "), aconteceu movimento de sintagma, enquanto, no segundo tipo ("O aluno ficou desmotivado"), não há movimento.

${ }^{9}$ Menuzzi (c.p.) observa que não parece haver elos não-locais em "João disse [que Pedro saiu]" e, mesmo assim, esta é uma estrutura de fraco desempenho entre os agramáticos. Apesar disso, deve-se notar que há duas fases na sentença em questão e a falta de localidade pode ser visualizada de outro modo. Além disso, parece haver pelo menos alguma relação entre os elementos da sentença matriz e os da encaixada, dada a distinção "*João disse [que Pedro saísse]" (não com o sentido de João ordenando a Pedro para sair, mas apenas relatando o fato de que Pedro saiu) e "João pediu [que Pedro saísse]".

${ }^{10}$ Em sua formulação mais recente, a estratégia não-lingüística se aplica somente a NPs [+animado] com referência própria; nos demais casos, a estratégia não se aplica, estando o acesso aos mecanismos internos do sistema lingüístico disponível para atribuição de papel temático.

${ }^{11}$ A Hipótese da Preservação de Elos Locais obedece a essa idéia, visto que a alteração que propõe (a indisponibilidade de elos não-locais) é mínima.

\section{REFERÊNCIAS BIBLIOGRÁFICAS}

BADECKER, D.; CARAMAZZA, A. On considerations of method and theory governing the use of clinical categories in neurolinguistics and cognitive neuropsychology: The case against agrammatism. Cognition 20, p. 97-125, 1985.

BAKER, M.; JOHNSON, K.; ROBERTS, I. Passive Arguments Raised. Linguistic Inquiry 20, p. 219-251, 1989.

BELLETTI, A.; RIZZI, L. Psych-verbs and Theta-theory. Natural Language and Linguistic Theory 6, p. 291-352, 1988.

BENNIS, H. Unergative adjectives and psych verbs. Manuscrito. 2000.

BERETTA, A.; HARFORD, C.; PATTERSON, J.; PIÑANGO, M. The derivation of post-verbal subjects: Evidence from aphasia. Natural Language and Linguistic Theory 1, p. 725-48, 1996.

BERNDT, R. S.; MITCHUM, C. C.; HAENDIGES, J. Comprehension of nonreversible sentences in "agrammatism": a meta-analysis. Cognition 58, p. 289308, 1996.

BOECKX, C. A minimalist view on the passive. University of Connecticut Occasional Papers, 2, 1998. 
CAPLAN, D. In defense of agrammatism. Cognition 24, p. 273-276, 1986.

. Agrammatism is a theoretically coherent aphasic category. Brain and Language 40, p. 274-281, 1991.

CAPLAN, D. Issues arising in contemporary studies of disorders of syntactic processing in sentence comprehension in agrammatic patients. Brain and Language 50, p. 325-338, 1995.

CAPLAN, D.; BAKER, C.; DEHAUT, F. Syntactic determinants of sentence comprehension in aphasia. Cognition 21, p. 117-75, 1985.

CARAMAZZA, A.; ZURIF, E. Dissociation of algorithmic and heuristic processes in language comprehension: evidence from aphasia. Brain and Language 3, p. 572-582, 1976.

CARAMAZZA, A.; CAPITANI, E.; REY, A., BERNDT, R. Agrammatic Broca's aphasia is not associated with a single pattern of comprehension. Brain and Language 76, p. 158-184, 2001.

CHOMSKY, N. Aspects of the theory of syntax. Cambridge, MA: MIT Press, 1965.

Knowledge of language. New York: Praeger, 1986.

. The minimalist program. Cambridge, MA: MIT Press, 1995.

. Minimalist Inquiries: The framework. In: MARTIN, R.; MICHAELS, D.; URIAGEREKA, J. (Ed.). Step by Step: Essays on Minimalist Syntax in Honor of Howard Lasnik. Cambridge, MA: MIT Press, p. 89-155, 2000.

. Derivation by phase. In: KENSTOWICZ, M. (Ed.), Ken Hale: A Life in Language, Cambridge, MA: MIT Press, p. 1-52, 2001.

DRAI, D., GRODZINSKY, Y. Comprehension regularity in Broca's aphasia: There's more of it than you ever imagined. Brain and Language 70, p. 139-43, 1999.

DRUKS, D., MARSHALL, D. Kicking over the Traces: A Note in Response to Zurif and Piñango. Brain and Language 75, p. 461-464, 2001.

FRIEDMANN, N.; GRODZINSKY, Y. Tense and agreement in agrammatic production: pruning the syntactic tree. Brain and Language 56, p. 397-425, 1997.

GRODZINSKY, Y. The syntactic characterization of agrammatism. Cognition 16, p. 99-120, 1984.

. On the interaction between linguistics and neuropsychology. Brain and Language 26, p. 186-196, 1985. 
GRODZINSKY, Y. Language deficits and the theory of syntax. Brain and Language 27, p. 135-159, 1986.

1990.

. Theoretical perspectives on language deficits. Cambridge, MA: MIT Press,

. There is an entity called agrammatic aphasia. Brain and Language 41, p. 555-564, 1991.

A restrictive theory of agrammatic comprehension. Brain and Language 50, p. $27-51,1995$.

. The neurology of syntax: language use without Broca's area. Behavioral and Brain Sciences 23, p. 1-71, 2000a.

Overarching agrammatism. In GRODZINSKY, Y.; SHAPIRO, L.; SWINNEY, D. (Ed.). Language and the brain. San Diego: Academic Press. p. 73-86, 2000b.

GRODZINSKY, Y.; FINKEL, L. The neurology of empty categories: aphasics' failure to detect ungrammaticality. Journal of Cognitive Neuroscience 10, p. 281-292, 1998.

HAGIWARA, H. The breakdown of functional categories and the economy of derivation. Brain and Language 50, p. 92-116, 1995.

HICKOK, G.; AVRUTIN, S. Representation, referentiality, and processing in agrammatic comprehension: two case studies. Brain and Language 50, p. 1026, 1995.

HICKOK, G.; ZURIF, E.; CANSECO-GONZALEZ, E. Structural description of agrammatic comprehension. Brain and Language 45, p. 371-395. 1993.

KEAN, M-L. The elusive character of agrammatism. Brain and Language 50, p. 369-384, 1995.

KITAHARA, H. Raising quantifiers without quantifier raising. In: ABRAHAM, W; EPSTEIN, S; THRÁINSSON, H; ZWART, J-W. Minimal ideas: syntactic studies in the minimalist framework. Amsterdam: John Benjamins, 1996.

KOLK, H.; VAN GRUNSVEN, M. On parallelism in agrammatism. In M.-L. Kean (Ed.) Agrammatism. New York: Academic Press, 1985.

LIMA, R. Hipótese da preservação de elos locais: uma explicação unificada para os déficits de compreensão e de produção no agramatismo. 2003. Tese (Doutorado em Lingüística) - Universidade Federal do Rio de Janeiro.

LINEBARGER, M. Agrammatism as evidence about grammar. Brain and Language 50, p. 52-91, 1995. 
LINEBARGER, M.; SCHWARTZ, M.; SAFFRAN, E. Sensitivity to grammatical structure in so-called agrammatic aphasics. Cognition 13, p. 361-392, 1983.

MARTIN, R. C.; WETZEL, W. F.; BLOSSOM-STACH, C.; FEHER, E. Syntactic loss versus processing deficit: An assessment of two theories of agrammatism and syntactic comprehension deficits. Cognition 32, p. 157-91, 1989.

MAUNER, G. Examining the empirical and linguistic bases of current theories of agrammatism. Brain and Language 50, p. 339-368, 1995.

MAUNER, G.; FROMKIN, V.; CORNELL, T. Comprehension and acceptability judgments in agrammatism: disruptions in the syntax of referential dependency. Brain and Language 45, p. 340-370, 1993.

MICELI, G.; MAZZUCCHI, A.; MENN, L.; GOODGLASS, H. Contrasting cases of English and Italian agrammatic aphasics. 1983. Apud SWINNEY D.; ZURIF E. Syntactic processing in aphasia. Brain and Language 50, p. 225-239, 1983.

NESPOULOUS, J.-L.; DORDAIN, M.; PERRON, C.; SKA, B.; BUB, D.; CAPLAN, D.; MEHLER, J.; LECOURS, A. Agrammatism in sentence production without comprehension deficits: Reduced availability of syntactic structures and/or of grammatical morphemes? Brain and Language 33, p. 273-295, 1988.

NOVAES, C. Formação de cadeias no agramatismo. Palavra 6, p. 166-174, 2000.

NUNES, J. Linearization of chains and sideward movement. Cambridge: MIT Press, 2004.

PESETSKY, D. Phrasal movements and its kin. Cambridge: MIT Press, 1998.

PIÑANGO, M. Some syntactic and semantic operations and their neurological underpinnings. Tese de Doutorado, Brandeis University, 1998.

RICHARDS, N. The principle of minimal compliance. Linguistic Inquiry 29, p. 599-631, 1998.

SADDY, D. Variables and events in the syntax of agrammatic speech. Brain and Language 50, p. 135-50, 1995.

SAFFRAN, E.; SCHWARTZ, M.: MARIN, O. The word order problem in agrammatism. II. Production. Brain and Language 10, p. 263-281, 1980.

SANCHEZ, M. Syntactic features in agrammatic production. 1996. Tese (Doutorado) - University of British Columbia.

SWINNEY D.; ZURIF E. Syntactic processing in aphasia. Brain and Language 50, p. 225-239, 1995. 
WEINBERG, A. A minimalist theory of human sentence processing. In: EPSTEIN, S.; HORNSTEIN, N. Working minimalism. Massachusetts: MIT Press. p.283-315. 1999.

WHITAKER, H. On the representation of language in the buman brain. Edmonton: Linguistic Research, 1970.

ZURIF, E.; PIÑANGO, M. The existence of comprehension patterns in Broca's aphasia. Brain and Language 70, p. 134-8, 1999. 